\title{
Exploring Catalyst Passivation with NMR Relaxation ${ }^{\dagger}$
}

Neil Robinson, ${ }^{\ddagger}$ Lynn F. Gladden and Carmine D’Agostino*

Department of Chemical Engineering and Biotechnology, University of Cambridge, Philippa Fawcett Drive, West

Cambridge Site, Cambridge, CB3 OAS, UK

¥ Presenting Author

* Corresponding Author. Email: cd419@cam.ac.uk

$\dagger$

Electronic Supporting Information (ESI) Provided 


\begin{abstract}
NMR relaxation has recently emerged as a novel and non-invasive tool for probing the surface dynamics of adsorbate molecules within liquid-saturated mesoporous catalysts. The elucidation of such dynamics is of particular relevance to the study and development of solvated green catalytic processes, such as the production of chemicals and fuels from bio-resources. In this paper we develop and implement a protocol using high field ${ }^{1} \mathrm{H}$ NMR spin-lattice relaxation as a probe of the reorientational dynamics of liquids imbibed within mesoporous oxide materials. The observed relaxation of liquids within mesoporous materials is highly sensitive to the adsorbed surface layer, giving insight into tumbling behaviour of spin-bearing chemical environments at the pore surface. As a prototypical example of relevance to liquid-phase catalytic systems, we examine the mobility of liquid methanol within a range of common catalyst supports. In particular, through the calculation and comparison of a suitable interaction parameter, we assess and quantify changes to these surface dynamics upon replacing surface hydroxyl groups with hydrophobic alkyl chains. Our results indicate that the molecular tumbling of adsorbed methanol is enhanced upon surface passivation due to the suppression of surface-adsorbate hydrogen bonding interactions, and tends towards that of the unrestricted bulk liquid. A complex analysis in which we account for the influence of changing pore structure and surface chemistry upon passivation is discussed. The results presented highlight the use of NMR spin-lattice relaxation measurements as a non-invasive probe of molecular dynamics at surfaces of interest to liquidphase heterogeneous catalysis.
\end{abstract}




\section{Introduction}

While many of the current experimental and theoretical developments across the field of heterogeneous catalysis concern themselves with the design, synthesis and optimisation of supported active species, ${ }^{1-3}$ the nature of the catalyst support itself can significantly influence catalytic reactions. For example, supports may directly influence the performance of supported metal species through strong metal-support interactions, ${ }^{4-6}$ including metal cluster anchoring ${ }^{7}$ and nanoparticle encapsulation, ${ }^{8,9}$ as well as direct participation in reaction pathways. ${ }^{10}$ Away from the active regions of the catalyst surface, the highly porous nature of typical catalyst supports also dictates mass transport properties through the existence of highly tortuous pore networks. ${ }^{11}$ Liquid-phase catalytic systems are typically of relevance to solvated green chemical processes. Here, the high molecular density of molecules containing polar functional groups may result in further mass transport limitations due to adsorption interactions at the support surface. ${ }^{11}$ While such surface interactions have been observed to enhance catalyst performance, ${ }^{12,13}$ this effect is also highly dependent on the nature of competitive adsorption between reagents and any solvent molecules present. ${ }^{14}$ One approach to limit unfavourable interactions between the support and mobile phase is to passivate the support surface via the covalent modification surface hydroxyl species. Indeed, the passivation of silica-based supports has been observed to enhance the performance of epoxidation, ${ }^{15,16}$ oxidation ${ }^{17}$ and hydrogenation catalysts. ${ }^{18-21}$ The influence of passivation also extends to other oxide materials; for example, Ellis et al. recently reported that passivation of $\gamma$-alumina leads to a $50 \%$ increase in activity for the dehydration of 1,2 -propanediol. ${ }^{22}$

In this work we wish to establish the use of liquid-phase nuclear magnetic resonance (NMR) relaxation as a surface sensitive method with which changes to adsobate mobility may be explored upon support passivation. The elucidation of such dynamics is of importance to the development of a range of liquid-phase catalytic processes. The successful functionalisation of catalyst surfaces has been extensively quantified using magic angle spinning solid-state NMR (see, for example refs $15,17,20$ ). However, the dynamic behaviour (diffusion, rotation) of liquid molecules imbibed within the pore network of functionalised catalysts has attracted far less attention. Pulsed-field gradient (PFG) NMR diffusometry has been used by Hansen ${ }^{23}$ and Courivaud ${ }^{24}$ to explore the influence of surface hydrophobicity on the self-diffusion of $n$ hexane imbibed within MCM-41. Elsewhere Weber et at. used PFG NMR analysis of Pd/ $\theta$-alumina saturated with 1-octene to illustrate how molecular self-diffusion with the adsorbed surface layer is inhibited through passivation of the pore surface. ${ }^{25}$

Herein, we describe the use of high field NMR spin-lattice relaxation measurements to probe changes in surface dynamics experienced by methanol - used here to represent a prototypical polar molecule of relevance to green chemical processes - within liquid-saturated catalyst supports which have been functionalised with alkane groups. 


\section{Surface Relaxation Theory}

Dipole-dipole interactions provide the primary contribution to NMR relaxation in the liquid phase. The dipolar spin-lattice relaxation rate for a collection of homonuclear spins $I$ within a static magnetic field of strength $B_{0}$ is given by the well-known equation ${ }^{26}$

$$
\frac{1}{T_{1}}=\left(\frac{\mu_{0}}{4 \pi}\right)^{2} \gamma_{I}^{2} \hbar^{2} \frac{3}{2} I(I+1)\left[J\left(\omega_{0}\right)+4 J\left(2 \omega_{0}\right)\right]
$$

where $\mu_{0}$ is the permeability of free space, $\gamma_{I}$ is the magnetogyric ratio of spin $I, \omega_{0}=\gamma_{I} B_{0}$ is the corresponding Larmor frequency and $T_{1}$ is the characteristic time constant for spin-lattice relaxation. $J(\omega)$ is the normalised spectral density function typically given by ${ }^{27}$

$$
J(\omega)=\frac{2 \tau_{c}}{1+\omega^{2} \tau_{c}^{2}}
$$

where the rotational correlation time $\tau_{c}$ describes the average time taken for spin-bearing molecules to rotate by 1 radian.

As a result of this relation, $T_{1}$ measurements may be used as an indicator of the rates of molecular tumbling within a given system; such an approach is often referred to as 'BPP Theory' after the seminal work in deriving this relation by Bloembergen, Purcell and Pound. ${ }^{28}$

In liquid-saturated mesoporous solids the observed relaxation rates are determined by the different liquid environments present. In this work we will assume the conditions of biphasic fast molecular exchange between an adsorbed surface layer of thickness $\delta$ and bulk liquid towards the centre of the pores, as described by Brownstein and Tarr. ${ }^{29,30}$ The observed relaxation rate $1 / T_{1, o b s}$ is a weighted average of bulk and surface contributions

$$
\frac{1}{T_{1, \text { obs }}}=\frac{1-P_{\text {surf }}}{T_{1, \text { bulk }}}+\frac{P_{\text {surf }}}{T_{1, \text { surf }}} .
$$

Here, $P_{\text {surf }}$ represents the proportion of spins (molecules) in the adsorbed surface layer and takes the form

$$
P_{\text {surf }}=\frac{\delta S}{V}
$$

where $S / V$ is the surface-to-volume ratio of the porous solid under study. Following Equations (1) and (2) the reduced molecular rotational mobility experienced by adsorbed species at the pore surface leads to $T_{1, \text { bulk }} \gg T_{1, \text { surf }}$. In combination with the high $S / V$ of liquids-saturated mesoporous media, the bulk term in Equation (3) becomes negligible, rendering relaxation measurements of such systems inherently surface sensitive. The observed relaxation rate is therefore often approximated to

$$
\frac{1}{T_{1, o b s}} \approx \frac{S}{V} \rho_{1}
$$

where $\rho_{1}=\delta / T_{1, \text { surf }}$ is known as the spin-lattice relaxivity. As a result, $T_{1}$ measurements performed on liquid-saturated mesoporous solids may be used to probe molecular mobility within the adsorbed surface layer; spin-lattice relaxation is a particularly useful probe of such dynamics as $T_{1}$ is independent of any internal magnetic field gradients which may occur at the solid-liquid interface under the influence of an applied magnetic field. ${ }^{31}$ Herein, we use this approach to compare the observed changes in methanol mobility across the surfaces of four common oxide support materials functionalised with octyl groups. 


\section{Experimental}

\subsection{Materials and Surface Passivation}

$\gamma$-Alumina $\left(\gamma-\mathrm{Al}_{2} \mathrm{O}_{3}\right), \theta$-alumina $\left(\theta-\mathrm{Al}_{2} \mathrm{O}_{3}\right)$ and silica $\left(\mathrm{SiO}_{2}\right)$ were obtained from Johnson Matthey, while anatase-titania $\left(\mathrm{A}-\mathrm{TiO}_{2}\right)$ was obtained from Evonik-Degussa. Each oxide was functionalised with surface octyl groups through liquid-phase treatment with triethoxy(octyl)silane (TEOS, Sigma Aldrich, $\geq 96 \%$ ). Oxide pellets were first dried at $105{ }^{\circ} \mathrm{C}$ for 2 hours before being soaked in excess TEOS for 12 hours. Pellets were then removed and washed several times in cyclohexane to remove any unreacted TEOS, before being dried at $105^{\circ} \mathrm{C}$ for a further 12 hours.

\subsection{Materials Characterisation}

Diffuse Reflectance Infrared Fourier Transform Spectroscopy (DRIFTS) measurements were performed to confirm successful passivation of the oxide supports. Measurements were performed on a ThermoFischer Nicolet iS50 FT-IR spectrometer equipped with a Praying Mantis diffuse reflectance cell and high temperature reaction chamber. Samples were ground by hand using a pestle and mortar and were analysed without dilution. Approximately $50 \mathrm{mg}$ of each catalyst support was loaded into the reaction cell supported by a small amount of quartz wool. To remove the influence of physisorbed water, all samples were heated to $150{ }^{\circ} \mathrm{C}$ at a rate of $10{ }^{\circ} \mathrm{C} \mathrm{min}{ }^{-1}$ under a low flow of helium; this temperature was maintained for 30 minutes after which samples were cooled to $25^{\circ} \mathrm{C}$ at the same rate. Spectra were acquired with 64 repeat scans with a resolution of $4 \mathrm{~cm}^{-1}$, relative to a $\mathrm{KBr}$ background acquired under the same conditions. Nitrogen isotherm measurements were performed using a Micromeritics TriStar 3000 automated gas adsorption analyser. Specific surface areas and were obtained by fitting to the Brunauer-Emmett-Teller (BET) equation, while pore volume and diameter values were calculated using the Barrett-Joyner-and-Halenda (BJH) method. All nitrogen adsorption measurements were carried out at $-195^{\circ} \mathrm{C}$.

\subsection{NMR Measurements}

Unfunctionalised oxides were dried for at least 12 hours at $105{ }^{\circ} \mathrm{C}$ before use. In order to ensure full saturation of the mesoporous structures each material was soaked in an excess of methanol (Sigma Aldrich, $\geq 99.8 \%$ ) for at least 24 hours. Separately, each material was also saturated with cyclohexane (Sigma Aldrich, $\geq 99.5 \%)$ in order to provide a weakly-interacting reference. The oxide materials were then removed from the liquid and rolled across a pre-soaked filter paper in order to remove any surface excess. Finally, the samples were transferred to sealed $5 \mathrm{~mm}$ NMR tubes for analysis. Each sample consisted of between 5 and 10 saturated catalyst pellets so as to provide a well-averaged measurement of the surfaceadsorbate interactions present between the imbibed liquids and the pore surfaces present throughout each oxide support.

${ }^{1} \mathrm{H}$ NMR spin-lattice relaxation measurements were performed using a Bruker DMX 300 spectrometer with a ${ }^{1} \mathrm{H}$ operational frequency of $300.13 \mathrm{MHz}$. All measurements were performed under ambient pressure at $20 \pm$ $0.2{ }^{\circ} \mathrm{C} . T_{1}$ values were acquired using the inversion-recovery method, ${ }^{27}$ during which 16 recovery delays were employed ranging logarithmically from $1 \mathrm{~ms}$ to $\sim 5 T_{1}$. For bulk liquids, $T_{1}$ values were be obtained by fitting the acquired data to

$$
\frac{M_{Z}(t)}{M_{0}}=1-2 \exp \left(\frac{-t}{T_{1}}\right)
$$


where $M_{z}(t)$ is the time-dependent longitudinal magnetisation and $M_{0}$ is the magnetisation at equilibrium.

From Equation (5) it is clear that for liquid-saturated mesoporous media, $T_{1}$ must form a distribution related to the spread of pore sizes throughout the solid. The acquired data may therefore be described by a Fredholm integral equation of the first kind. ${ }^{32}$

$$
\frac{M_{z}(t)}{M_{0}}=\int_{-\infty}^{\infty} K\left(t, T_{1}\right) F\left(T_{1}\right) d \log \left(T_{1}\right)+\varepsilon
$$

The kernel function $K\left(t, T_{1}\right)=1-2 \exp \left(-t / T_{1}\right)$ describes the predicted form of $T_{1}$ relaxation and $\varepsilon$ represents the experimental noise. For each sample analysed the required $T_{1}$ distribution $F\left(T_{1}\right)$ was obtained via a numerical inversion of Equation (7). ${ }^{32}$ Stability of the inverted distributions in the presence of noise was achieved through the use of Tikhonov regularisation, ${ }^{33}$ with the degree of smoothing chosen using the Generalised Cross Validation method. ${ }^{34}$ As the shape and width of the resulting distributions are highly susceptible to both experimental noise and the signal-to-noise ratio of the acquired data, we take only the modal values $\left\langle T_{1, o b s}\right\rangle$ from these distributions in the following analysis. Further details are provided in the Electronic Supporting Information (ESI).

\section{Results and Discussion}

\subsection{Surface Chemistry and Pore Structure}

We first examine the influence of passivation on the surface chemistry and pore structure of the mesoporous oxides studied. Figure 1 shows the difference in infrared adsorption spectra between passivated and unpassivated oxides. It is clearly apparent that functionalisation with TEOS results in a loss of surface hydroxyl groups, characterised by the negative peaks present above $3500 \mathrm{~cm}^{-1}$. Indicated are the frequencies and corresponding assignments for the peaks which appear upon passivation. Four stretches are observed around $2900 \mathrm{~cm}^{-1}$ corresponding to $-\mathrm{CH}_{2}\left(2926 \mathrm{~cm}^{-1}\right.$ asymmetric and $2855 \mathrm{~cm}^{-1}$ symmetric) and $\mathrm{CH}_{3}\left(2961 \mathrm{~cm}^{-1}\right.$ asymmetric and $2879 \mathrm{~cm}^{-1}$ symmetric) stretches. ${ }^{35}$ An additional peak is observed at $1465 \mathrm{~cm}^{-1}$ ${ }^{1}$ which we assign to a $-\mathrm{CH}_{2}$ bending mode. ${ }^{35}$ The presence of these peaks confirms qualitatively the successful passivation of surface hydroxyls with alkyl groups upon treatment of our oxides with TEOS. 


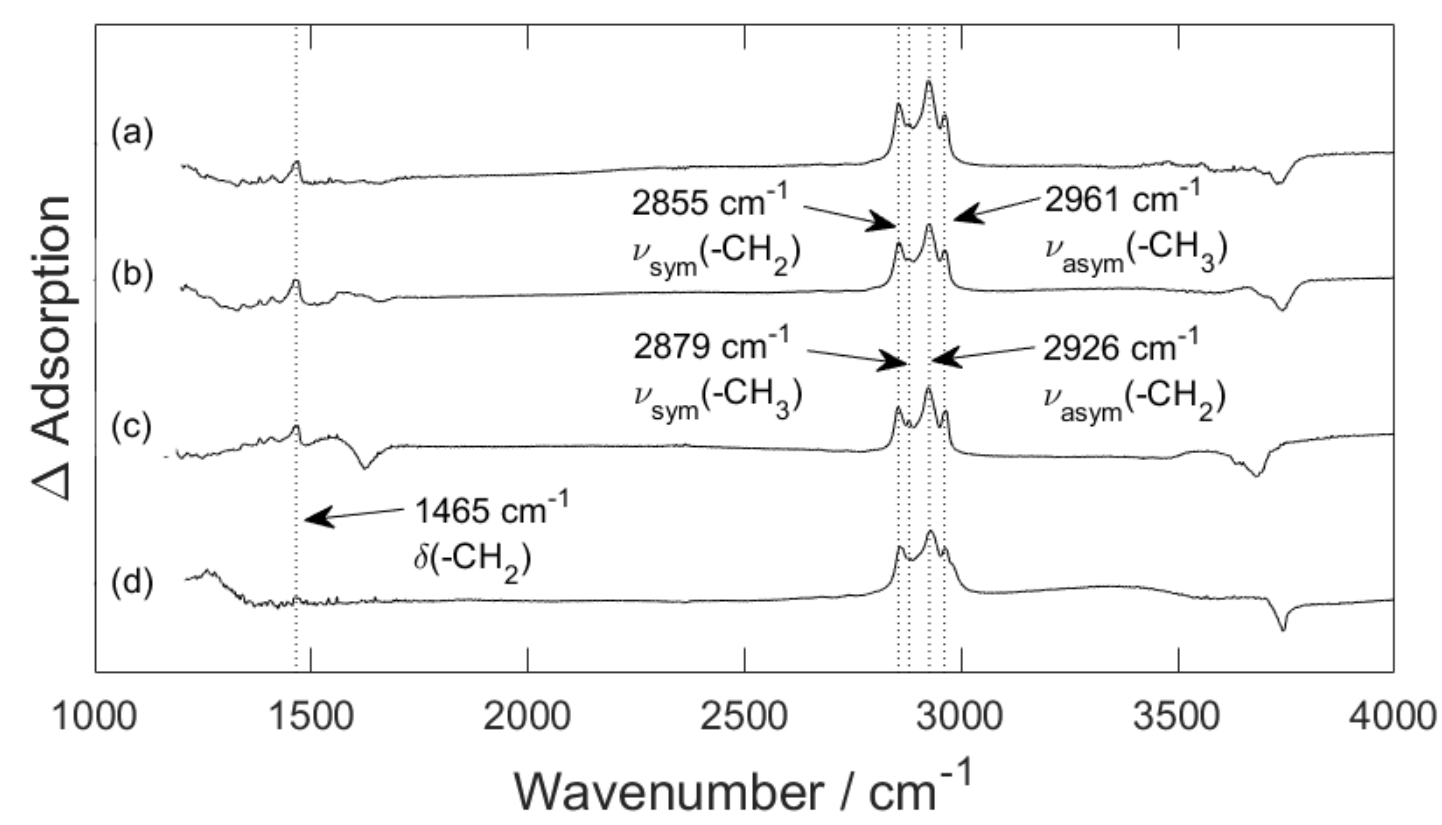

Figure 1. DRIFTS spectra for a) $\gamma$-Alumina, b) $\theta$-Alumina, c) A-Titania and d) Silica. Spectra are reported as change in absorption upon passivation of the oxide surface with TEOS. Negative peaks therefore represent a loss of signal upon surface functionalisation while positive peaks indicate a gain in signal upon functionalisation.

To examine the effect of passivation on the pore structure of our oxides we have performed specific surface area and pore volume measurements pre- and post-functionalisation. Results are provided in Table 1 and indicate a reduction in both surface area and pore volume upon passivation. The surface-to-volume ratio $(S / V)$ of each material calculated from these results is also given, indicating an increase in $S / V$ upon passivation.

Table 1. BET and BJH analysis for passivated (oxide+TEOS) and unpassivated mesoporous oxide supports.

\begin{tabular}{c|cc|cc|cc}
\multirow{2}{*}{ Oxide Support } & \multicolumn{2}{|c|}{ BET Surface Area $\left(\mathbf{m}^{\mathbf{2}} \mathbf{g}^{-\mathbf{1}}\right)$} & \multicolumn{2}{c|}{ BJH Pore Volume $\left(\mathbf{c m}^{\mathbf{3}} \mathbf{g}^{-\mathbf{1}}\right)$} & \multicolumn{2}{c}{$\mathbf{S} / \mathbf{~ V ~}\left(\mathbf{m}^{\mathbf{2}} \mathbf{c m}^{-\mathbf{3}}\right)$} \\
\cline { 2 - 7 } & Oxide & Oxide+TEOS & Oxide & Oxide+TEOS & Oxide & Oxide+TEOS \\
\hline $\mathrm{Y}-\mathrm{Al}_{2} \mathrm{O}_{3}$ & 90 & 82 & 0.49 & 0.34 & 184 & 244 \\
$\theta-\mathrm{Al}_{2} \mathrm{O}_{3}$ & 108 & 99 & 0.57 & 0.52 & 188 & 189 \\
$\mathrm{~A}-\mathrm{TiO}_{2}$ & 40 & 37 & 0.28 & 0.19 & 142 & 196 \\
$\mathrm{SiO}_{2}$ & 272 & 236 & 1.29 & 1.01 & 211 & 235
\end{tabular}




\subsection{NMR Spin-Lattice Relaxation}
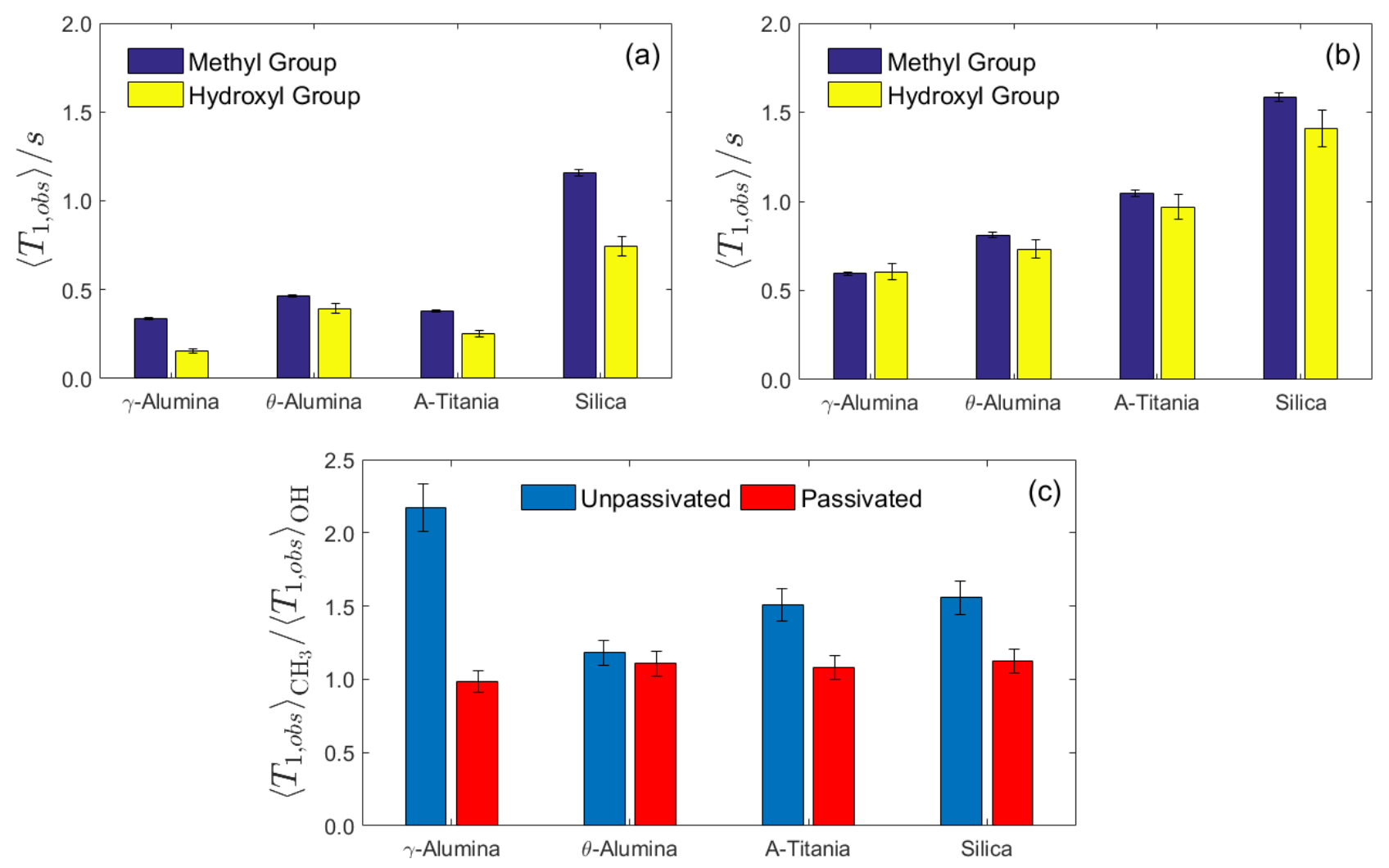

Figure 2. $\left\langle T_{1, o b s}\right\rangle$ values for methanol imbibed within a) unpassivated and b) passivated oxides, together with c) the ratio of $\left\langle T_{1, o b s}\right\rangle$ exhibited by the methyl $\left(\mathrm{CH}_{3}\right)$ and hydroxyl $(\mathrm{OH})$ proton environments within each material.

The acquired $\left\langle T_{1, o b s}\right\rangle$ values characterising the observed rates of longitudinal proton relaxation within our methanol-saturated oxides are presented in the top two sections of Figure 2. Figure 2a displays the relaxation time constants within unpassivated oxides. It is evident from these data that the methyl group presents a longer $\left\langle T_{1, o b s}\right\rangle$ time than exhibited by the methanol hydroxyl groups imbibed within the same support. This is consistent across the range of oxides investigated and may be explained in terms of the relative motional freedom of the two proton environments. According to the BPP theory of nuclear spin relaxation, as $\tau_{c} \rightarrow 0$, i.e. as tumbling becomes more rapid, $T_{1} \rightarrow \infty$. A longer relative $T_{1}$ may therefore be recognised as an increase in observed tumbling rate, and hence motional freedom at the pore surface. The reduced relative motional freedom exhibited by hydroxyl groups within the adsorbed surface layer is easily explained through their ability to form hydrogen bonds with other polar moieties, resulting in favourable adsorption interactions with the hydroxyl groups which decorate the pore surfaces within unpassivated oxides. Hydroxyl protons therefore experience reduced mobility at the pore surface relative to the apolar methyl group, leading to more rapid $\left\langle T_{1, o b s}\right\rangle$ times. Other surface interactions may also cause a bias towards a reduced $T_{1}$ of the hydroxyl group, such as the reversible dissociation of labile hydroxyl protons from adsorbed methanol molecules onto the pore surface, and interactions with acidic metal sites.

Figure $2 \mathrm{~b}$ displays the same data for methanol-saturated oxides which have been passivated. We observe through a comparison of Figures $2 \mathrm{a}$ and $2 \mathrm{~b}$ that the difference in $T_{1}$ experienced by the two proton environments is noticeably reduced upon passivation. We may quantify this change by taking the ratio of observed relaxation time constants, $\left\langle T_{1, o b s}\right\rangle_{M e} /\left\langle T_{1, o b s}\right\rangle_{O H}$. This is a simple analysis, and may be performed without any consideration of the changes to pore structure or surface chemistry upon passivation. This is 
because the terms $\mathrm{S} / \mathrm{V}$ and $\delta$ within Equation (5) cancel exactly when considering molecules within the same pore structure. Values obtained by taking this ratio are displayed in Figure $2 \mathrm{c}$. In the case of bulk methanol (ESI Figure S2), the methyl group was found to have $T_{1, M e}=4.22 \pm 0.01 \mathrm{~s}$ while the hydroxyl group was found to have $T_{1, O H}=3.92 \pm 0.01 \mathrm{~s}$. The resulting ratio of relaxation time constants in the bulk is therefore $T_{1, M e} / T_{1, O H} \approx 1$. From consideration of the ratios presented in Figure $2 \mathrm{c}$ it appears that, within our passivated oxides in general, the ratio of relaxation time constants tends towards that of the bulk liquid. In turn, this indicates that bulk-like liquid dynamics occur near the surface of passivated pores, whereas the increased ratio of relaxation time constants observed within unpassivated supports indicates a more significant difference in the mobility of the two functional groups. Such a difference is of course expected following the above discussion on hydrogen bonding interactions with unpassivated surfaces.

\subsection{Influence of Passivation on Relaxation Time Interpretation}

It is also apparent from Figure 2 that $\left\langle T_{1, o b s}\right\rangle$ increases upon passivation regardless of the proton environment considered. From the above discussion we may be tempted to suggest that this is an obvious indication of enhanced molecular freedom within the adsorbed surface layer of passivated oxides. However, from Equation (5) it is clear that in order to compare the observed tumbling behaviour of molecules within different materials, we must also take into account any changes to the surface-to-volume (S/V) ratio of the pores within each material, as well as the influence of passivation on the thickness of the adsorbed surface layer, $\delta$.

In this section, we therefore wish to provide an alternative method of analysing our relaxation data, in which the influence of passivation on pore structure and oxide surface chemistry is taken into account. We also aim to provide a more general method of analysing changes to molecular mobility at pore surfaces, in which the presence of multiple spin-bearing environments is not a prerequisite. We begin by defining a dimensionless 'spin-lattice interaction parameter' $\hat{\eta}(\delta)$, given by the ratio of $T_{1}$ in the bulk liquid to that of the same liquid at the pore surface.

$$
\hat{\eta}(\delta)=\frac{T_{1, \text { bulk }}}{T_{1, \text { surf }}(\delta)}
$$

Such a parameter provides a convenient limiting value of $\hat{\eta}(\delta)=1$, in which case molecular rotational mobility within the adsorbed surface layer is considered identical to that within the bulk. Adsorption interactions leading to a reduction in surface mobility will therefore be expressed as an increase in $\hat{\eta}(\delta)$ from this limit. In previous work we have made use of a similar metric $\eta=T_{1, b u l k} / T_{1, o b s}$ to compare the surface dynamics of polar liquids within a range of unfunctionalised catalyst supports. ${ }^{11}$ However, such an approach would be inappropriate here as it must be assumed that no change occurs to the thickness of the adsorbed surface layer upon passivation. In the current analysis we avoid this assumption by replacing the observed relaxation time constant with that associated with relaxation directly at the surface; $T_{1, \operatorname{surf}}(\delta)$ is obtained through a simple rearrangement of Equation (3) such that

$$
T_{1, \text { surf }}(\delta)=P_{\text {surf }}\left[\frac{1}{\left\langle T_{1, \text { obs }}\right\rangle}-\frac{\left(1-P_{\text {surf }}\right)}{T_{1, \text { bulk }}}\right]^{-1} .
$$


A dependence on the unknown surface layer thickness $\delta$ arises from the presence of the surface population term $P_{\text {surf }}$. S/V - also present within $P_{\text {surf }}$ - is estimated through the nitrogen absorption analysis discussed previously. Values are given in Table 1, from which it is clear that treatment of the oxide supports with TEOS causes an increase in $S / V$ across the range of oxides considered. From examination of the approximate relation between $T_{1, o b s}$ and $S / V$ provided by Equation (5), and by assuming a constant surface layer thickness and $T_{1, \text { surf }}$ across both functionalised and unfunctionalised oxides, it is instructive to note that such a change should lead to a reduction in $\left\langle T_{1, o b s}\right\rangle$ upon functionalisation, rather than the observed increase. Our results, therefore, cannot be explained through the inclusion of $S / V$ alone. Indeed, it is necessary that the change in surface relaxivity $\left(\delta / T_{1, \text { surf }}\right)$ which occurs as a result of surface passivation, outweighs the influence of increasing S/V.

\subsection{Analysis of Methyl Group Dynamics}

We chose to analyse our spin-lattice interaction parameter across an adsorbed surface thickness range of $\delta=1-5$ molecular layers. This is an appropriate range for the analysis of alcohols interacting with oxide surfaces. For example, molecular dynamics simulations of methanol adsorbing at a model silica surface suggest that a surface layer thickness of 2 molecular layers is readily formed. ${ }^{36}$ Experimental studies of the adsorption of ethanol at silica suggest a similar coverage. ${ }^{37}$ We define the thickness of a single adsorbed layer within a given liquid-saturated pore to be that of the kinetic diameter of the molecule under study, $k_{d}$. For methanol, it is therefore assumed that $1 \delta=d_{k} \approx 3.6 \AA$ A during our relaxation experiments. ${ }^{38}$

To simplify the analysis of methanol dynamics, and to generate a method dependent on the presence of only a single relaxation environment, the $T_{1}$ relaxation of the methyl group is considered an appropriate representation of molecular mobility within our oxides. The spin-lattice relaxation of alkyl protons has previously been applied as a measure of overall molecular mobility in a number of liquid-saturated mesoporous oxides. ${ }^{11}$ Indeed, in comparison to the relaxation characteristics of the hydroxyl group, relaxation within the methyl environment presents an attractive measure of molecular dynamics. Not only does it contain a significantly higher fraction of the total spins present within a given methanol-saturated pore, but its relaxation is also independent of any proton hopping, or other dissociative dynamics exhibited by the hydroxyl group. Indeed, we note that while the rotational mobility of methyl protons about the C-O axis of a given methanol molecule will be relatively unaffected by the interaction of the hydroxyl group with the pore surface, the rates of tumbling about axes parallel to the pore surface will still be significantly reduced, leading to sensitivity in changes to the overall molecular tumbling motion upon adsorption.

The calculated rotational interaction parameters for each of the methanol-saturated oxides investigated are presented in Figure 3. On examination of these results it is clear that functionalisation of the pore surface has a significant impact on $\hat{\eta}(\delta)$. More specifically, passivation of the surface hydroxyl groups results in a notable decrease in $\hat{\eta}(\delta)$, irrespective of oxide material or surface layer thickness. From Equation (8) it is clear that this is indicative of an increase in the tumbling mobility of methanol molecules within the adsorbed surface layer. In particular, our results indicate that the motional freedom of methanol molecules within the adsorbed surface layer tends towards that of the unrestricted bulk liquid upon interaction with passivated pore surface. As this change is accompanied by a decrease in surface hydroxyl density upon functionalisation, we may attribute this observation to a reduction in the hydrogen bonding ability of the oxide surfaces upon passivation. Importantly, it should be noted that this result is qualitatively identical to 
that proposed by the simple analysis in Section 4.2, however, here we have performed a far more extensive assessment, involving the evaluation and elimination of the effects of changing $\mathrm{S} / \mathrm{V}$ and $\delta$ values.

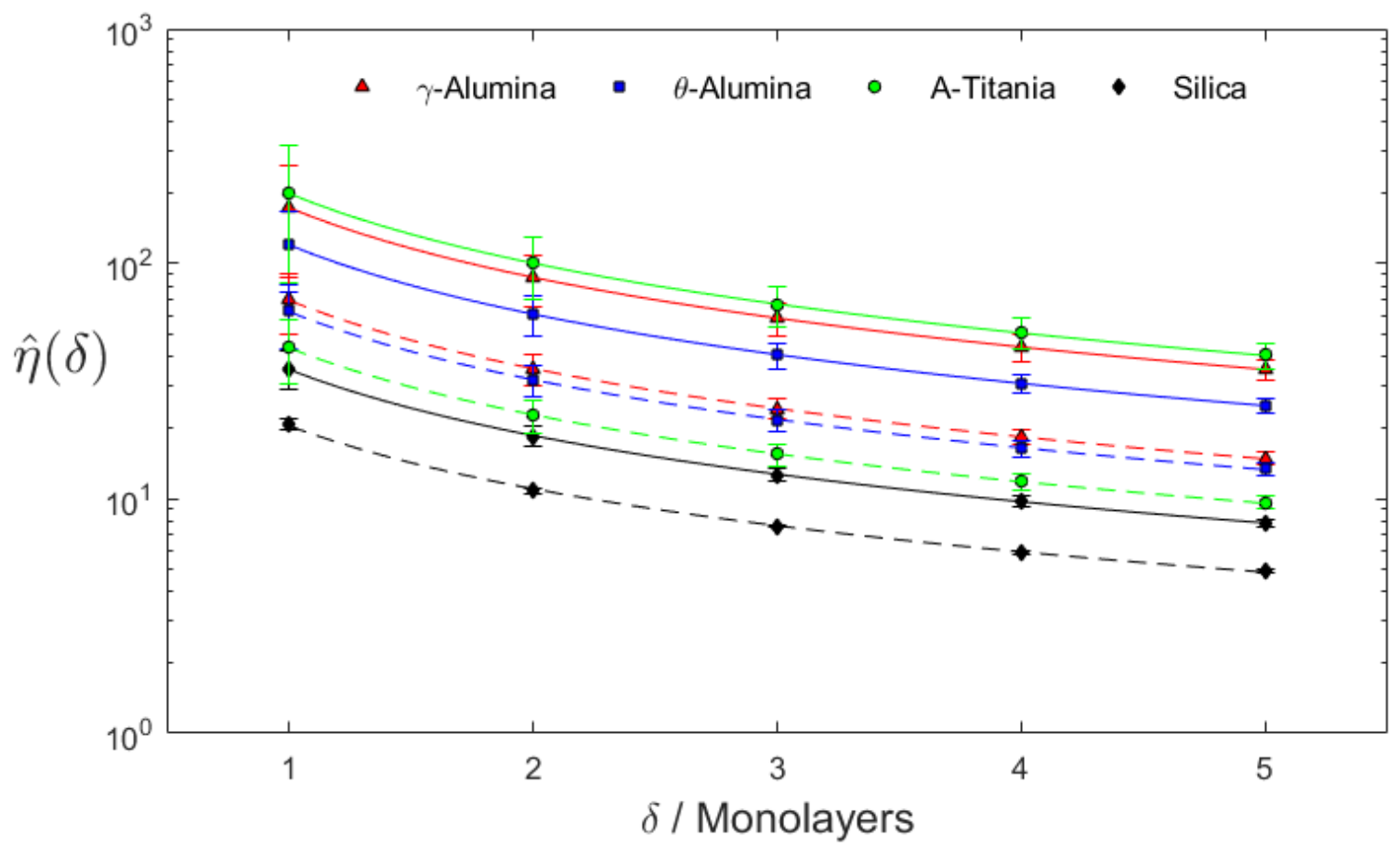

Figure 3. Rotational surface interaction parameter as a function of surface layer thickness for methanol saturated mesoporous oxides. A fit to the data according to the power law discussed in the text is shown in each case, where solid lines indicate unpassivated oxides and dashed lines indicate those passivated with TEOS.

\subsection{Derivation of a Surface Layer Independent Interaction Parameter}

It is interesting to observe that the data presented in Figure 3 fit a power law of the form $\hat{\eta}_{i}(\delta)=a_{i} \delta^{-b_{i}}$. Here $a_{i}\left(m^{b_{i}}\right)$ and $b_{i}$ (dimensionless) are constants and the index $i$ indicates the oxide under study. Moreover, this simple relationship suggests an approximate method to remove the dependency of $\hat{\eta}(\delta)$ on $\delta$. We attempt to achieve this through the normalisation of $\hat{\eta}(\delta)$ against a weakly-interacting reference liquid. We first define a corresponding 'normalised' spin-lattice interaction parameter as

$$
\hat{\eta}_{i}^{N}=\frac{\hat{\eta}_{i}^{M e}\left(\delta_{j}\right)}{\hat{\eta}_{i}^{C H X}\left(\delta_{j}\right)}
$$

where $\hat{\eta}_{i}^{C H X}\left(\delta_{j}\right)$ is the spin-lattice interaction parameter acquired from cyclohexane-saturated oxides. Cyclohexane $\left(d_{k} \approx 6.2 \AA\right)^{38}$ is often chosen as a weakly-interacting reference species for NMR relaxation and diffusion studies involving heterogeneous catalysts due to its single proton environment, slow relaxation characteristics and low affinity for oxide surfaces. ${ }^{11-13,39,40}$ Within the oxide materials investigated here cyclohexane also exhibits the same power law relationship between $\hat{\eta}(\delta)$ and $\delta$ as observed for methanol. We note from Equation (10) and from our empirical power law relation, that in order for the dependency on $\delta$ to be removed, $b$ values acquired from the fitting of methanol and cyclohexane interaction parameter data must satisfy the condition $b_{i}^{M e}-b_{i}^{C H X} \approx 0$. In turn, the respective magnitudes of $\hat{\eta}_{i}^{N}$ will be 
proportional to the ratio $a_{i}^{M e} / a_{i}^{C H X}$. The power law constants $a$ and $b$ are listed in Table 2 for both methanol- and cyclohexane-saturated oxides. It is clear from these data, and by consulting the above condition on the magnitude of relative $b$ values, that in order to remove the dependency on $\delta$, the methanol relaxation data pertaining to both passivated and unpassivated oxides must be normalised by the relaxation behaviour of cyclohexane in unpassivated oxides alone. Indeed, the combination of these $b$ values provides the closest match to the above condition on $b$ values for the four oxides considered, while $b$ values obtained from cyclohexane-saturated oxides passivated with TEOS are significantly different to those obtained for methanol in either passivated or unpassivated materials.

Table 2. $a$ and $b$ values for passivated (oxide+TEOS) and unpassivated mesoporous oxide supports saturated with either methanol and cyclohexane. These constants correspond to the power law fit $\hat{\eta}_{i}(\delta)=a_{i} \delta^{-b_{i}}$ where $i$ indicates the identity of the oxide.

\begin{tabular}{|c|c|c|c|c|c|c|c|c|}
\hline \multirow{3}{*}{$\begin{array}{l}\text { Oxide } \\
\text { Support }\end{array}$} & \multicolumn{4}{|c|}{ Methanol } & \multicolumn{4}{|c|}{ Cyclohexane } \\
\hline & \multicolumn{2}{|c|}{ Oxide } & \multicolumn{2}{|c|}{ Oxide+TEOS } & \multicolumn{2}{|c|}{ Oxide } & \multicolumn{2}{|c|}{ Oxide+TEOS } \\
\hline & $a / m^{b}$ & $\boldsymbol{b}$ & $a / m^{b}$ & $\boldsymbol{b}$ & $a / m^{b}$ & $\boldsymbol{b}$ & $a / m^{b}$ & $\boldsymbol{b}$ \\
\hline $\mathrm{V}-\mathrm{Al}_{2} \mathrm{O}_{3}$ & $8.42 \times 10^{-8}$ & 0.99 & $5.23 \times 10^{-8}$ & 0.97 & $6.11 \times 10^{-8}$ & 0.95 & $6.06 \times 10^{-7}$ & 0.76 \\
\hline$\theta-\mathrm{Al}_{2} \mathrm{O}_{3}$ & $6.66 \times 10^{-8}$ & 0.98 & $5.08 \times 10^{-8}$ & 0.96 & $7.58 \times 10^{-8}$ & 0.91 & $3.11 \times 10^{-7}$ & 0.80 \\
\hline $\mathrm{A}-\mathrm{TiO}_{2}$ & $9.32 \times 10^{-8}$ & 0.99 & $4.96 \times 10^{-8}$ & 0.95 & $8.14 \times 10^{-8}$ & 0.90 & $1.51 \times 10^{-6}$ & 0.70 \\
\hline $\mathrm{SiO}_{2}$ & $5.18 \times 10^{-8}$ & 0.94 & $7.64 \times 10^{-8}$ & 0.89 & $4.10 \times 10^{-7}$ & 0.78 & $6.79 \times 10^{-7}$ & 0.75 \\
\hline
\end{tabular}

The indices $j$ within Equation (10) highlight the fact that the values of $\delta$ considered must be equal in units of length. Despite the differing values of $d_{k}$ for methanol and cyclohexane, the correct values of $\hat{\eta}_{i}^{C H X}(\delta)$ are readily obtained by consulting the appropriate power law constants governing the behaviour of cyclohexane (ESI Figure S20) and solving for the corresponding methanol $\delta$ values.

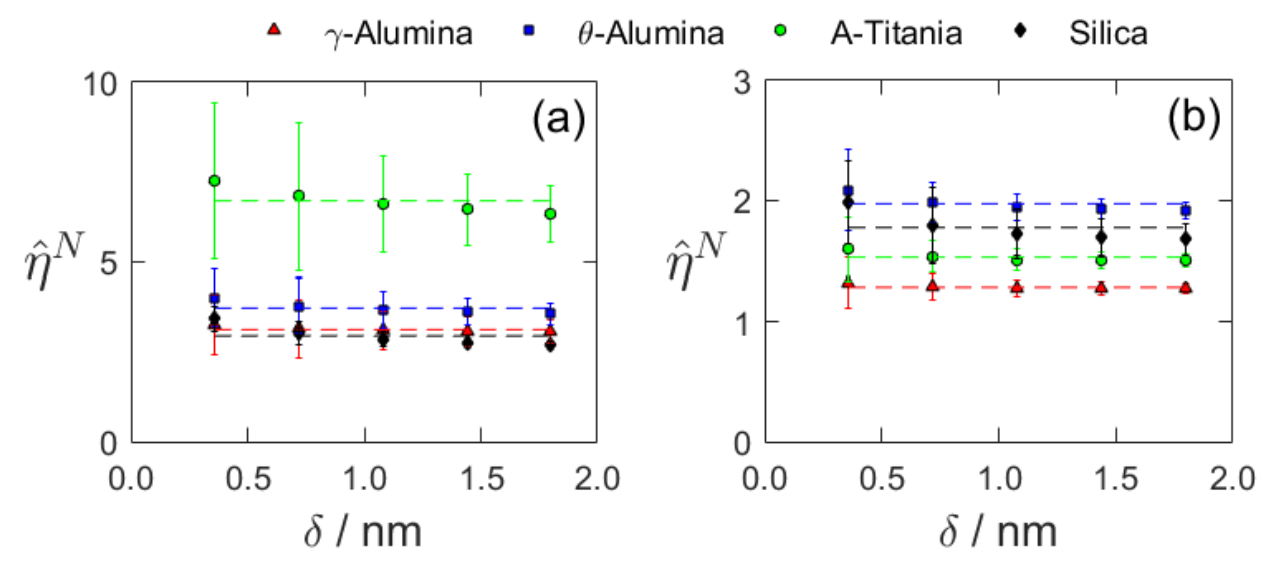

Figure 4. Normalised rotational interaction parameters for methanol within a) unpassivated and b) passivated oxides, as a function of surface layer thickness.

The normalised spin-lattice interaction parameters for methanol imbibed within unpassivated and passivated oxides are shown in Figures $4 a$ and $4 b$, respectively. From these data we may deduce that, within the experimental error indicated for each oxide, the dependency on the surface layer thickness has indeed been removed through the normalisation process detailed above. As a result we obtain a single, $\delta$ independent value of $\eta^{N}$ for each material by averaging the acquired $\eta^{N}$ values across the range of $\delta$ values investigated. In unpassivated oxides these are 3.1, 3.7, 6.7 and 3.0 for $\gamma$-alumina, $\theta$-alumina, anatase-titania and silica, respectively. Upon passivation these values reduced to $1.3,2.0,1.5$ and 1.8 , respectively. It is 
again apparent that passivation of the surface hydroxyls within each mesoporous oxide has significantly enhanced the molecular tumbling of liquid methanol at the pore surface, indicating a reduction in surfaceadsorbate interactions upon removal of surface hydroxyl groups. Furthermore, it is significant to note that while our previous methods of evaluating changes to the motional freedom of imbibed methanol concentrate on the comparison of the imbibed liquid behaviour relative to that of the unrestricted bulk, this analysis compares the dynamics of imbibed methanol to that of an imbibed reference. More specifically, as $\hat{\eta}_{i}^{N} \rightarrow 1$ the molecular tumbling rates of methanol tend towards that of our cyclohexane reference, when imbibed within unpassivated oxide materials. This is deemed to be an adequate, non-interacting reference system, as interactions between cyclohexane and hydroxylated oxide surfaces are known to be negligible. ${ }^{11}$

\section{Summary and Conclusions}

In this work we have demonstrated the use of NMR spin-lattice relaxation measurements as a non-invasive probe of molecular dynamics within liquid-saturated mesoporous oxides. Such measurements are particularly sensitive to the tumbling motion of molecules within the adsorbed surface layer. These measurements have been applied in order to assess changes in the surface dynamics of methanol upon passivation of surface hydroxyl groups. Our results show that passivation significantly increases the motional freedom of adsorbed liquid methanol. We have presented an extensive protocol designed to analyse in detail changes to the tumbling dynamics of adsorbed methanol upon passivation. By taking the ratio of observed spin-lattice relaxation time constants of the two proton environments present, and by comparing the results to that obtained from unrestricted bulk liquid, we have shown that passivation of the oxide supports cause methanol mobility in the adsorbed surface layer to tend towards that of the bulk. A more complex analysis based on the rotational dynamics of the methyl group alone leads to the same conclusion. This method is based on the calculation of a dimensionless interaction parameter and considers the influence of changes in surface chemistry and pore structure upon passivation. As such, this method not only provides a rigorous assessment of changes to alcohol dynamics within these oxides, but may also be extended, in future work, to more general cases of surface functionalisation. Finally, we have proposed an approximate method to remove any influence of the surface layer thickness on our results, based on the normalisation of methanol dynamics by a weakly-interaction reference system. Overall, this work illustrates NMR spin-lattice relaxation measurements as a useful probe of adsorbate dynamics within systems of relevance to liquid-phase catalysis. In particular, the calculation of a relevant interaction parameter and its comparison to simpler methods of mobility analysis enhances our ability to interpret the results of spinlattice relaxation measurements in terms of modified surface affinity within functionalised mesoporous materials.

\section{Acknowledgements}

N.R. would like to thank the Catalysis@Cambridge initiative for a studentship. C.D. would like to acknowledge the support of Wolfson College Cambridge. Mr Zlatko Saracevic is also gratefully acknowledged for performing the pore size measurements. 


\section{References}

1 C. H. Christensen and J. K. Nørskov, J. Chem. Phys., 2008, 128, 182503.

2 F. Calle-Vallejo, J. Tymoczko, V. Colic, Q. H. Vu, M. D. Pohl, K. Morgenstern, D. Loffreda, P. Sautet, W. Schuhmann and A. S. Bandarenka, Science, 2015, 350, 185-189.

3 A. A. Latimer, A. R. Kulkarni, H. Aljama, J. H. Montoya, J. S. Yoo, C. Tsai, F. Abild-Pedersen, F. Studt and J. K. Nørskov, Nat. Mater., 2017, 16, 225-229.

4 J. C. Matsubu, S. Zhang, L. DeRita, N. S. Marinkovic, J. G. Chen, G. W. Graham, X. Pan and P. Christopher, Nat. Chem., 2017, 9, 120-127.

5 J. A. Farmer and C. T. Campbell, Science, 2010, 329, 933-936.

6 S. J. Tauster, Acc. Chem. Res., 1987, 20, 389-394.

7 R. Burgess, C. Buono, P. R. Davies, R. J. Davies, T. Legge, A. Lai, R. Lewis, D. J. Morgan, N. Robinson and D. J. Willock, J. Catal., 2015, 323, 10-18.

8 M. Bowker and R. Sharpe, Catal. Struct. React., 2015, 1, 140-145.

9 O. Dulub, W. Hebenstreit and U. Diebold, Phys. Rev. Lett., 2000, 84, 3646-3649.

10 J. Graciani, K. Mudiyanselage, F. Xu, A. E. Baber, J. Evans, S. D. Senanayake, D. J. Stacchiola, P. Liu, J. Hrbek, J. F. Sanz and J. A. Rodriguez, Science, 2014, 345, 546-550.

11 C. D'Agostino, J. Mitchell, L. F. Gladden and M. D. Mantle, J. Phys. Chem. C, 2012, 116, 8975-8982.

12 C. D'Agostino, G. L. Brett, P. J. Miedziak, D. W. Knight, G. J. Hutchings, L. F. Gladden and M. D. Mantle, Chem. - Eur. J., 2012, 18, 14426-14433.

13 C. D’Agostino, T. Kotionova, J. Mitchell, P. J. Miedziak, D. W. Knight, S. H. Taylor, G. J. Hutchings, L. F. Gladden and M. D. Mantle, Chem. - Eur. J., 2013, 19, 11725-11732.

14 C. D’Agostino, M. R. Feaviour, G. L. Brett, J. Mitchell, A. P. E. York, G. J. Hutchings, M. D. Mantle and L. F. Gladden, Catal. Sci. Technol., 2016, 6, 7896-7901.

15 K. Lin, L. Wang, F. Meng, Z. Sun, Q. Yang, Y. Cui, D. Jiang and F.-S. Xiao, J. Catal., 2005, 235, 423-427.

16 M. Guidotti, I. Batonneau-Gener, E. Gianotti, L. Marchese, S. Mignard, R. Psaro, M. Sgobba and N. Ravasio, Microporous Mesoporous Mater., 2008, 111, 39-47.

17 N. Igarashi, K. Hashimoto and T. Tatsumi, Microporous Mesoporous Mater., 2007, 104, 269-280.

18 M. Ojeda, F. J. Pérez-Alonso, P. Terreros, S. Rojas, T. Herranz, M. López Granados and J. L. G. Fierro, Langmuir, 2006, 22, 3131-3137.

19 L. Jia, L. Jia, D. Li, B. Hou, J. Wang and Y. Sun, J. Solid State Chem., 2011, 184, 488-493.

20 A. S. Zola, L. S. da Silva, A. L. Moretti, A. do C. Fraga, E. F. Sousa-Aguiar and P. A. Arroyo, Top. Catal., 2016, 59, 219-229.

21 N. A. Fellenz, J. F. Bengoa, M. V. Cagnoli and S. G. Marchetti, J. Porous Mater., 2016, 1-12.

22 L. D. Ellis, S. Pylypenko, S. R. Ayotte, D. K. Schwartz and J. W. Medlin, Catal. Sci. Technol., 2016, 6, 57215728.

23 E. W. Hansen, F. Courivaud, A. Karlsson, S. Kolboe and M. Stöcker, Microporous Mesoporous Mater., 1998, 22, 309-320.

24 F. Courivaud, E. W. Hansen, A. Karlsson, S. Kolboe and M. Stöcker, Microporous Mesoporous Mater., 2000, 35-36, 327-339.

25 D. Weber, A. J. Sederman, M. D. Mantle, J. Mitchell and L. F. Gladden, Phys. Chem. Chem. Phys., 2010, 12, 2619-2624.

26 A. Abragam, The Principles of Nuclear Magnetism, Clarendon Press, 1961.

27 M. H. Levitt, Spin Dynamics: Basics of Nuclear Magnetic Resonance, Wiley-Blackwell, Chichester, England ; Hoboken, NJ, 2nd Edition., 2008.

28 N. Bloembergen, E. M. Purcell and R. V. Pound, Phys. Rev., 1948, 73, 679-712.

29 K. R. Brownstein and C. E. Tarr, J. Magn. Reson. 1969, 1977, 26, 17-24.

30 K. R. Brownstein and C. E. Tarr, Phys. Rev. A, 1979, 19, 2446-2453.

31 K. E. Washburn, C. D. Eccles and P. T. Callaghan, J. Magn. Reson., 2008, 194, 33-40.

32 J. D. Wilson, J. Mater. Sci., 1992, 27, 3911-3924.

33 A. N. Tikhonov and V. I. Arsenin, Solutions of ill-posed problems, Winston, 1977.

34 G. Wahba and Y. Wang, Commun. Stat. - Theory Methods, 1990, 19, 1685-1700. 
35 F. Scheinmann, An Introduction to Spectroscopic Methods for the Identification of Organic Compounds: Nuclear Magnetic Resonance and Infrared Spectroscopy, Elsevier, 2013.

36 D. Roy, S. Liu, B. L. Woods, A. R. Siler, J. T. Fourkas, J. D. Weeks and R. A. Walker, J. Phys. Chem. C, 2013, 117, 27052-27061.

37 D. Wu, X. Guo, H. Sun and A. Navrotsky, J. Phys. Chem. C, 2015, 119, 15428-15433.

38 H. Wu, Q. Gong, D. H. Olson and J. Li, Chem. Rev., 2012, 112, 836-868.

39 C. D’Agostino, M. R. Feaviour, G. L. Brett, J. Mitchell, A. P. E. York, G. J. Hutchings, M. D. Mantle and L. F. Gladden, Catal. Sci. Technol., 2016.

40 S. N. Lanin, E. V. Vlasenko, N. V. Kovaleva and F. T. Zung, Russ. J. Phys. Chem. Focus Chem., 2008, 82, 2152-2155. 